\title{
Aerobic Exercise Improves Attention and Quality of Sleep Among Professional Volleyball Players
}

\author{
Morteza Taheri ${ }^{1^{*}}$, Fariba Valayi ${ }^{2}$ \\ ${ }^{1}$ Assistant Professor of sport sciences, Imam Khomeini International University, Qazvin, Iran \\ ${ }^{2}$ MS Student of Sport Sciences, Islamic Azad University, Karaj, Iran
}

\begin{abstract}
Low quality of sleep leading to cognitive problems is among key a reasons that declines the athlete performance especially due to stressful athletic events. Thus, the aim of the study was to investigate the effect of aerobic Exercise on attention, and quality of sleep among professional volleyball players. 21 professional female volleyball players were selected through purposive sampling and divided randomly into two experimental $(n=11$; with mean age of 19.3 years old) and control group ( $n=10$; with mean age of 19.6 years old). Subjects were measured for attention performance and quality of sleep using Vienna system test and Pittsburgh Sleep Quality Index (PSQI) respectively. The exercise protocol included an eight-week aerobic exercise, three times per week, and 60 minutes per session with $60 \%-70 \%$ of the maximum heart rate. The intensity of exercise protocol was monitored by Polar Electro. Dependent and Independent t-test were used to analyze the data. The results suggested that both attention tests (speed processing and selective attention) were improved significantly in posttest in experimental group compared to control group $(p \leq 0.05)$. In addition, It was shown that, quality of sleep was significantly improved in experimental group ( $p=0.001 ; t=8.01$ ). Although professional athletes often do athletic activities, the regular aerobic exercise would improve their psychomotor performance and quality of sleep. Hence, it can be considered as a noninvasive and effective strategy to counteract sleep loss consequences and attention deficits caused by stressful conditions in athletic events.

Keywords: Attention, Quality of sleep, Athlete
\end{abstract}

\section{INTRODUCTION}

High quality sleep is vital for optimum sporting performance of professional and elite athletes in that a great deal of physiological and psychological processes occur during sleep such as mental relaxation, clearance of

\footnotetext{
*Correspondence: m.taheri@soc.ikiu.ac.ir

Morteza Taheri, Department of Physical Education, Faculty of Social

Sciences, am Khomeini International University

Phone: 989124070721 Fax: 982833901606
}

Received: 20 March 2018 Accepted: 01 April 2018

\section{Sleep and Hypnosis \\ Journal homepage: \\ www.sleepandhypnosis.org \\ ISSN: 2458-9101 (Online)}

brain metabolites and restoration of nervous, immune, skeletal, and muscular systems (Kline, 2014). In this regard, One of major concerns of coaches for improving their athletes performance is how to recovery their physiological and mental status (Yousef Abadi, Mirzaei, Habibi, \& Barbas, 2017). Regarding the necessity of athletic preparation for events, having provided the relaxation conditions leading to an optimal sleep is another issue which must be taken into consideration. Hence, knowing about the important factors which positively affect athletic performances is a topic of great interest among researchers. There is a great deal of evidences showing that athletes suffer from sleep deprivation due to stressful conditions they have. This 
deteriorating condition affects negatively cognitive, motor functioning and psychomotor performance such as reaction time and memory tasks (Monleon, Hemmati Afif, Mahdavi, \& Rezayi, 2018; Taheri \& Arabameri, 2012). On the other hand, The adverse effect of sleep deprivation on athletic performance especially those needed attention has been well documented (Fullagar et al., 2015; Thun, Bjorvatn, Flo, Harris, \& Pallesen, 2015).

There are several studies supporting the notion that any factor affecting sleep adversely influences attention, simple and choice reaction time significantly (Philip et al., 2003). Indeed, the crucial decision regarding the effects of the exercise on both quality of sleep and psychomotor performance has not been reported. It must be noted that, the effect of sleep on athletic performance has been much studied compared to vice versa (Kline, 2014). With respect to the effect of exercise on attention and sleep, different results can be found. In a study, it was shown that quality of sleep isn't affected by exercise (Wunsch, Kasten, \& Fuchs, 2017), while there are some research supporting the positive effects of exercise on quality of sport (K. Irandoust \& Taheri, 2017; Khadijeh Irandoust \& Taheri, 2018). Given the deleterious effects of stressful conditions on quality of sleep and attention capacity, it was aimed at identifying the effect of regular aerobic exercises on quality of sleep and attention performance in professional female volleyball players.

\section{METHODS}

The study was a semi experimental research with pretest and post-test design. 21 professional female volleyball players who were suffered from sleep loss [score $\geq 5$ Pittsburgh Sleep Quality Index (PSQI)] were selected voluntarily and divided randomly into two experimental ( $n=11$; with mean age of 19.31 years old) and control group ( $n=10$; with mean age of 19.64 years old). Exclusion criteria included: lack of injury within the past 6 month; no history of attention deficit based on their self-reports. Attention performance and quality of sleep were measured by Vienna system test and PSQI respectively (Khadijeh Irandoust \& Taheri, 2018). There were two subtest for measuring attention in subjects including speed processing and selective attention. The research design is seen in table 1 . The research was approved by the Ethics Committee of Imam Khomeini international university (ref no. 17628). Written informed consent was obtained from all subjects. The study was conducted in accordance with the ethical standards of the Helsinki Declaration (1964).

Table 1. Research design

\begin{tabular}{|c|c|c|c|c|}
\hline Groups & Phase & pretest & Intervention (aerobic exercises) & Post-test \\
\hline \multicolumn{2}{|c|}{ Experimental } & Attention Test & $\checkmark$ & Attention Test \\
\hline \multicolumn{2}{|c|}{ Control } & Sleep Monitoring & - & Sleep Monitoring \\
\hline
\end{tabular}

Table 2. Comparing the attention Performance before and after intervention

\begin{tabular}{|c|c|c|c|c|c|c|c|c|c|c|}
\hline \multicolumn{2}{|c|}{ Tests } & \multirow{3}{*}{$\begin{array}{c}\text { Group } \\
\text { Number of } \\
\text { Correct } \\
\text { Rejection }\end{array}$} & \multicolumn{2}{|c|}{ Control } & \multirow{2}{*}{$\mathrm{t}$} & \multirow{2}{*}{$\mathrm{P}$} & \multicolumn{2}{|c|}{ Experimental } & \multirow{2}{*}{$t$} & \multirow{2}{*}{$\mathrm{P}$} \\
\hline \multirow{4}{*}{ 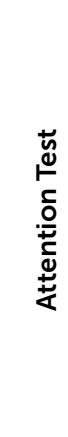 } & \multirow{2}{*}{$\begin{array}{l}\text { Processing } \\
\text { Speed }\end{array}$} & & Pretest & Post test & & & Pretest & Post test & & \\
\hline & & & $21.35 \pm 0.9$ & $21.2 \pm 1.0$ & 0.63 & 0.32 & $21.42 \pm 1.0$ & $19.48 \pm 0.44$ & 6.35 & $0.001^{*}$ \\
\hline & \multirow{2}{*}{$\begin{array}{l}\text { Selective } \\
\text { Attention }\end{array}$} & $\begin{array}{c}\text { Mean } \\
\text { Time for } \\
\text { Correct } \\
\text { Responses }\end{array}$ & $1.86 \pm 0.07$ & $1.87 \pm 0.05$ & -1.77 & 0.13 & $1.86 \pm 0.10$ & $1.77 \pm 0.08$ & -7.41 & $0.001^{*}$ \\
\hline & & $\begin{array}{c}\text { Mean } \\
\text { Time for } \\
\text { Correct } \\
\text { Rejections }\end{array}$ & $1.88 \pm 0.05$ & $1.87 \pm 0.07$ & -0.81 & 0.43 & $1.89 \pm 0.08$ & $1.77 \pm 0.06$ & -6.90 & $0.001^{*}$ \\
\hline
\end{tabular}


The exercise protocol consisted of an eight-week aerobic exercise, three sessions a week (each session, 60 minutes). The intensity of exercise protocol was monitored by Polar Electro, Kempele, Finland (60-70\% MaxHR). The exercise protocol included three major parts: 1- warm-up (10 minutes with 30-40\% MaxHR); 2- main program (40 minutes of aerobic exercises with 30-40\% MaxHR); 3- cool-down (10 minutes of light exercises with 10-20\% MaxHR) (Jafari, Pouryamehr, \& Fathi, 2017). Normality distribution of variables was tested using Kolmogorov Smirnov test. The data were analyzed by Dependent and independent t- tests using statistical package version 21 (SPSS Inc., Chicago, IL).

\section{RESULTS}

The results can be seen in table 2 and figure 1. Paired t-test results suggested that processing speed (number of correct rejection) ( $p=0.001)$ and selective attention (mean time for correct responses and rejections) as attention tests were improved significantly in posttest in experimental group compared to control group $(p=0.001)$.

According to independent t-test results, no significant difference was found between the two groups in pretest $(p \leq 0.05)$, while in post-test, experimental group had a significant improvement in both tests $(p \geq 0.05)$. As shown in figure 1 , aerobic exercise improved the quality of sleep in professional female athletes $(p=0.001 ; t=8.01)$.

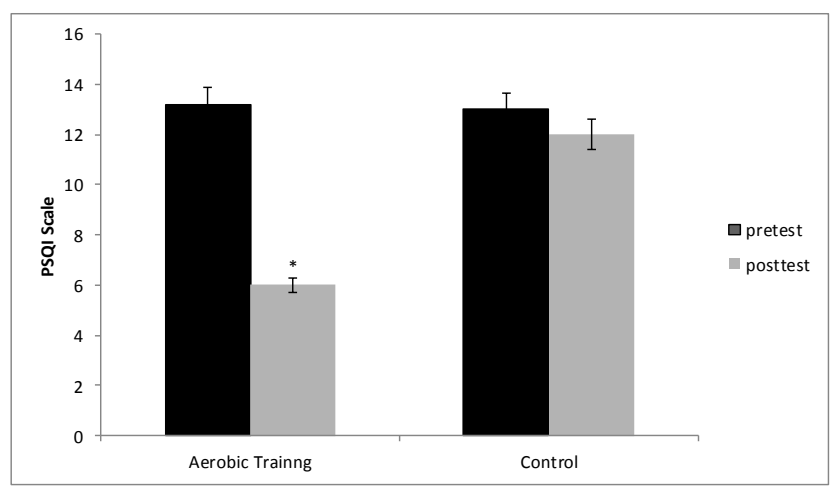

Figure 1. The effects of aerobic training on quality of sleep in professional volleyball players

\section{DISCUSSION}

The research was designed in such a way that any difference in attention and sleep status of subjects could be attributed to the exercise protocol. Two key findings were found in the study. First, aerobic exercise has positive influences on concentration and speed processing factors as attention indices which play key roles in successful performance in decisive tasks such as volleyball. This results is consistent with Monleon, et al (2018), who reported that aerobic exercise would improve the sleep cycles in athletes (Monleon et al., 2018) while others had different results (Lo, Ong, Leong, Gooley, \& Chee, 2016). One reason for getting conflicting findings may be the exercise intensity, volume and type and different assessments and age groups. Scientific evidences show that the prefrontal cortex is extremely affected by sleep loss. On the other hand, elite athletes are more vulnerable to sleep loss due to competitive stress and heavy exercises. Therefore, it seems logical that aerobic exercise alleviate fatigue status in athletes and provide them in relaxation condition and all these leading to a more optimal executive function such as better attention and sleep (Harrison, Horne, \& Rothwell, 2000). Additionally, our findings shed more light to the issue that volleyball players should involve more in aerobic activities compared to strength and plyometric exercises. Since, getting well mentally prepared and also having good attention and sleep cycle are of utmost importance for achieving optimal athletic performance. So, assessing and monitoring the physical, physiological and psychological characteristics must be performed periodically (Pavlovic et al., 2018; Reza Sharif \& Sayyah, 2018; Sayyah, Rahimi, Bigdeli, \& Rajabi, 2011). Conclusively, our results demonstrate that aerobic exercises results in better attention and sleep, hence, it can be considered as a noninvasive and effective strategy to counteract cognitive impairment caused by stressful conditions in athletic events. These results along with some other research (K. Irandoust \& Taheri, 2017; Taheri \& Irandoust, 2017) raise the possibility that doing the aerobic exercise may be one component of increasing cognitive performance. It must be noted that larger sample size with longitudinal designs 
and recruiting more frequent subjective assessments as well as objective assessments are highly recommended in future studies. New techniques for monitoring sleep quality (e.g. Polysomnography) can be applied for a more exact conclusion.

Acknowledgments: Special thanks to all volleyball players of Qazvin medical science university who generously devote their valuable time to the study.

Conflict of interest: The authors declare no conflict of interest.

Informed consent: Informed consent was obtained from all the participants included in the study.

Funding: The authors declare that the current study was not financially supported by any institution or organization.

\section{References}

Fullagar, H. H., Skorski, S., Duffield, R., Hammes, D., Coutts, A. J., \& Meyer, T. (2015). Sleep and athletic performance: the effects of sleep loss on exercise performance, and physiological and cognitive responses to exercise. Sports medicine, 45(2), 161186.

Harrison, Y., Horne, J. A., \& Rothwell, A. (2000). Prefrontal neuropsychological effects of sleep deprivation in young adults--a model for healthy aging? Sleep, 23(8), 1067-1073.

Irandoust, K., \& Taheri, M. (2017). The Effect of Vitamin D supplement and Indoor Vs Outdoor Physical Activity on Depression of Obese Depressed Women. Asian J Sports Med, 8(3), e13311. doi:10.5812/asjsm.13311

Irandoust, K., \& Taheri, M. (2018). The Effect of Strength Training on Quality of Sleep and Psychomotor Performance in Elderly Males. Sleep and Hypnosis, 20(3), 160-165.

Jafari, M., Pouryamehr, E., \& Fathi, M. (2017). The Effect of Eight Weeks High Intensity Interval Training (HIIT) on E-Selection and P-Selection in Young Obese Females. Int J Sport Stud HIth, In Press(In Press), e64336. doi:10.5812/intjssh.64336

Kline, C. E. (2014). The bidirectional relationship between exercise and sleep: Implications for exercise adherence and sleep improvement. American journal of lifestyle medicine, 8(6), 375-379. doi:10.1177/1559827614544437

Lo, J. C., Ong, J. L., Leong, R. L., Gooley, J. J., \& Chee, M. W. (2016). Cognitive Performance, Sleepiness, and Mood in Partially Sleep Deprived Adolescents: The Need for Sleep Study. Sleep, 39(3), 687-698. doi:10.5665/sleep.5552

Monleon, C., Hemmati Afif, A., Mahdavi, S., \& Rezayi, M. (2018). The Acute Effect of Low Intensity Aerobic Exercise on Psychomotor Performance of Athletes with Nocturnal Sleep Deprivation. Int J Sport Stud Hith, In Press(In Press), e66783. doi:10.5812/intjssh.66783

Pavlovic, R., Mihajlovic, I., Idrizovic, K., Vrcic, M., Stankovic, D., \& Joksimovic, M. (2018). Differences in Anthropometric Traits and Trend of Changes in High School Students. Int J Sport Stud Hlth, In Press(In Press), e68101. doi:10.5812/intjssh.68101

Philip, P., Sagaspe, P., Taillard, J., Moore, N., Guilleminault, C., Sanchez-Ortuno, M., . . Bioulac, B. (2003). Fatigue, sleep restriction, and performance in automobile drivers: a controlled study in a natural environment. Sleep, 26(3), 277280.

Reza Sharif, M., \& Sayyah, M. (2018). Assessing Physical and Demographic Conditions of Freshman Male Medical Students. Int J Sport Stud HIth, In Press(In Press), e67421. doi:10.5812/ intjssh.67421

Sayyah, M., Rahimi, S., Bigdeli, M., \& Rajabi, M. (2011). Comparing the anthropometric characteristics of injured and non-injured girl student athletes participating in the sport olympiads held by the ministry of health and medical education in the summer of 2009 in the city of Yazd. Biosciences Biotechnology Research Asia, 8(2), 367-371.

Taheri, M., \& Arabameri, E. (2012). The effect of sleep deprivation on choice reaction time and anaerobic power of college student athletes. Asian journal of sports medicine, 3(1), 15.

Taheri, M., \& Irandoust, K. (2017). The Exercise-Induced Weight Loss Improves Self-Reported Quality of Sleep in Obese Elderly Women with Sleep Disorders. Sleep and Hypnosis. doi, 10.

Thun, E., Bjorvatn, B., Flo, E., Harris, A., \& Pallesen, S. (2015). Sleep, circadian rhythms, and athletic performance. Sleep medicine reviews, 23, 1-9.

Wunsch, K., Kasten, N., \& Fuchs, R. (2017). The effect of physical activity on sleep quality, well-being, and affect in academic stress periods. Nature and Science of Sleep, 9, 117-126. doi:10.2147/NSS.S132078

Yousef Abadi, H. A., Mirzaei, B., Habibi, H., \& Barbas, I. (2017). Prevalence of Rapid Weight Loss and Its Effects on Elite Cadet Wrestlers Participated in the Final Stage of National Championships. Int J Sport Stud HIth, In Press(In Press), e64316. doi:10.5812/intjssh.64316 